\title{
Carpal tunnel release by mini palmar incision
}

\author{
Kawee Pataradool, Pravit Kitidumrongsook, Adisorn Patradul \\ Department of Orthopaedics, Faculty of Medicine, Chulalongkorn University, Bangkok, 10330, \\ Thailand
}

\begin{abstract}
Background: Open carpal tunnel release is the gold standard treatment for carpal tunnel syndrome. However, there are complications related to the long incision.

Method: We report the results from a mini-incision open carpal tunnel release with simple instruments.

Results: There were no complications in our small series, improvement of scores was shown at four-week follow-up, and cosmetic results were satisfactory.

Conclusions: Mini-incision carpal tunnel release is one option to treat carpal tunnel syndrome.
\end{abstract}

Keywords: Boston carpal tunnel questionnaire, carpal tunnel syndrome, conventional open carpal tunnel release, mini-incision carpal tunnel release

Carpal tunnel syndrome (CTS) is the most common entrapment neuropathy of upper extremities. Open carpal tunnel release (OCTS) is the most frequent surgical procedure and the gold standard for cases that do not respond to conservative treatment [1].The traditional surgical approach uses a longitudinal palmar incision from the wrist across the palmar aspect of the hand. It provides direct vision of the ligament, guarantees complete section, and allows treatment of additional pathologies. Its major drawbacks are an unsightly and tender scar, pain, a long healing period, and cosmetic complaints. All of which are related to the long incision [2, 3].

In an effort to decrease these complications, endoscopic carpal tunnel release (ECTR) was developed in 1989 to leave the skin intact over the transverse carpal ligament (TCL) and eliminate the problems of traditional OCTR $[4,5]$. The disadvantage of ECTR includes costly equipment, prolonged operative setup time, and a technically demanding procedure. Most importantly there have been many

Correspondence to: Kawee Pataradool, MD, Department of Orthopaedics, Faculty of Medicine, Chulalongkorn University, 1873 Rama IV, Pathumwan, Bangkok 10330 Thailand. E-mail: kaweeet@hotmail.com reported complications associated with ECTR, including transaction of the median nerve or its branches and incomplete division of TCL. [5-7].

Several authors tried to combine the simplicity and safety of OCTR with the less tissue trauma and reduced post-operative morbidity of ECTR by using a short incision and open technique. These included a wrist crease mini-incision [8-12], short longitudinal palmar incision [13-16], and double mini-incision [17]. With these approaches, TCL is classified based on whether CTS is carried out with a conventional instrument or specially developed ones.

We report our early experience and results with a longitudinal mini incision over the palm using simple instruments.

\section{Materials and Methods Surgical technique}

The operations were performed under local infiltration anesthesia at the palm along the thenar crease. A fore arm rubber band tourniquet was used as shown in Fig.1.

A $1.5 \mathrm{~cm}$ longitudinal incision was placed $0.5 \mathrm{~cm}$ proximal to a transverse line from the ulnar side of the abducted thumb, bisecting the axis of the ring finger, or ulnar to thenar crease. The palmar fascia was 
divided in line with the skin incision. The deep transverse carpal ligament (TCL) was identified, and incised with blade as a small hole. After that, a small blunt tip clamp was passed through this hole from proximal to distal direction for protection of underlying median nerve. A blade was placed over the clamp and used to release distal edge of TCL under direct vision to avoid injury to thenar branch of the median nerve and the vascular arch. Wherever proximal edge was divided by blunt tip scissors, a blind technique from ulnar to palmaris longus to avoid injury to palmar cutaneous branch of median nerve was used. The skin was closed with 5-0 Nylon using about three stitches and a compression dressing was applied. Then, the tourniquet was released.

Between March and June 2008, we performed 11 carpal tunnel releases on 11 patients. The diagnosis of CTS was clinical and confirmed by electrodiagnostic studies on all patients. All the patients had failed prior conservative treatment for at least one month before surgery.
A

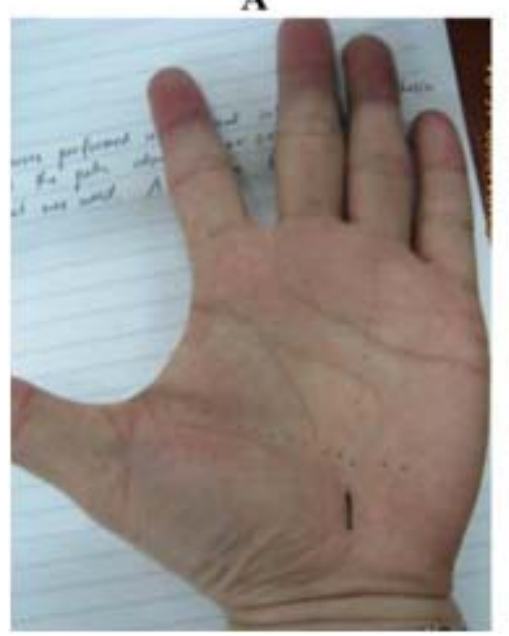

C

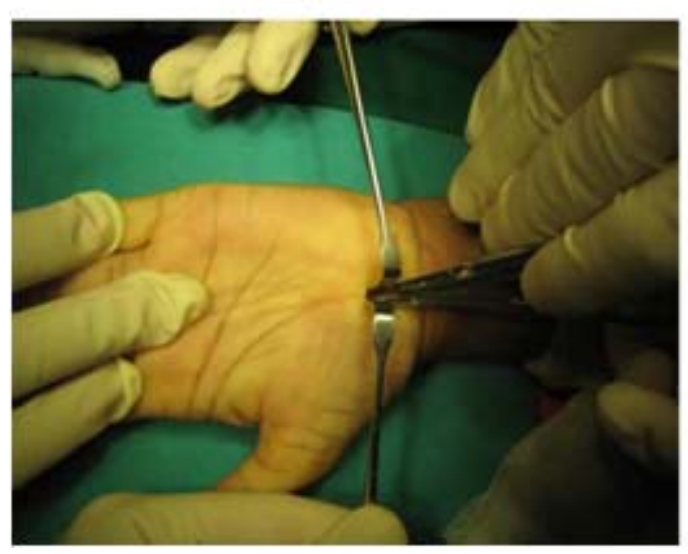

B

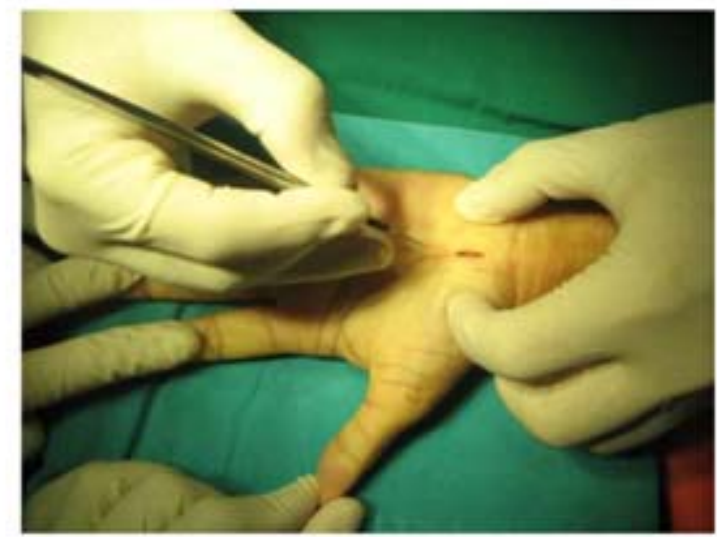

D

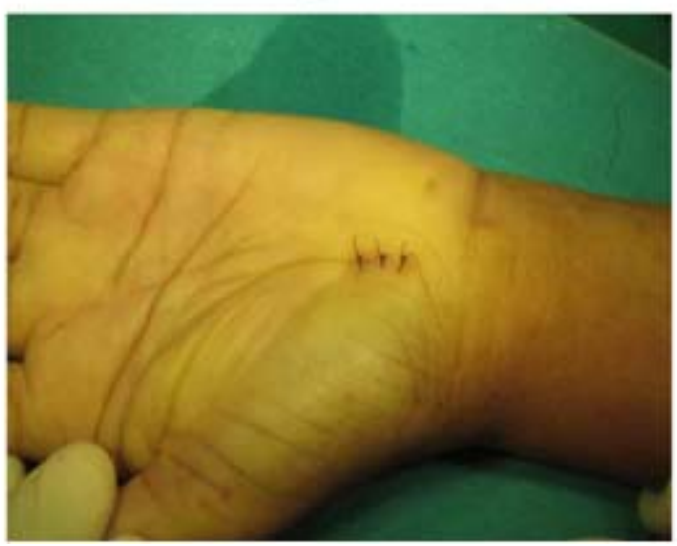

Fig. 1 The operation stages. A: Surgical site marked; B: Surgical wound opened by scapel; C: Small blunt tip clamp inserted for median nerve guard and blade used for divided distal edge of TCL; D: Three stitch skin closed with 5-0 nylon. 
Table 1. Demographic data of patients.

\begin{tabular}{ll}
\hline Number of patients & 11 \\
Female:Male & $9: 2$ \\
Age (years) & Mean 51.36 (range 28-61) \\
Right hands:Left hands & $7: 4$ \\
Average duration of symptoms & 10.8 months (3-24 months)
\end{tabular}

Surgical results and outcomes were evaluated using the Boston Carpal Tunnel questionnaire (BCT) developed and tested by Levine in 1993 [18]. BCT questionnaire is a patient oriented, self-administered standardized instrument that has shown reproducibility and validity [19]. It consists of two scales. The symptom severity scale includes 11 items concerning severity, frequency, and duration of symptoms. The functional status scale includes eight items concerning difficulties in performing eight daily activities. The symptom severity and functional status scores are the mean scores of all items in each scale ranging from one (no symptom functional status) to five (most severe symptom and functional status). The cosmetic results were rated by the patients as poor, fair, good, or excellent.

\section{Results}

The preoperative and four-week post operative scores for symptom severity and functional status scales of the BCT are shown in Table 2.

Both scales showed large improvements of scores before surgery compared with four weeks after surgery compared with symptom and functional status scores before surgery. The cosmetic results were rated as excellent in nine patients and good in two patients. There were no major complications. Only three patients reported scar pain.

\section{Discussion}

Conventional open carpal tunnel release has been widely accepted as an effective method for treating carpal tunnel syndrome after failed conservative management [1]. However, the incision is long and in some instances, it heals with an unsightly scar and cosmetic complaints [2, 3]. Endoscopic carpal tunnel release seems to decrease morbidity from conventional technique, but still have some problems [4-7]. More recently, various open techniques using small incisions have been described by either conventional instruments or special instruments.

Carter (1991) used a transverse wrist incision and a special carpal tunnel knife and reported no complication in 100 cases [8]. Some authors have used a short palmar incision for carpal tunnel surgery. Bromley (1994) used short palmar incision and released carpal tunnel with scissors and blind technique [14]. Lee and Strickland (1998) used the same incision but used a special knife to release carpal tunnel [16].

Our procedure used conventional instruments for carpal tunnel release with a longitudinal mini-incision in the palm. We cut distal edge of TCL by direct vision to avoid thenar branch of median nerve and cut proximal edge by scissors with blind technique but stay on ulnar side of palmaris longus tendon to avoid injury to palmar cutaneous branch of median nerve because this nerve always lies on the radial side of palmaris longus tendon [15, 19]. Boston selfadministered questionnaire was used for assessment the results. This questionnaire has excellent reproducibility, and is widely used for subjective assessment for the results of CTS management [18, $20,21]$. Our results show improvement in symptom severity scores and functional status scores in four weeks after surgery compared to before surgery. The cosmetic results are very good according to patient satisfaction. There are no major complications noted in our patients.

Table 2. The Boston carpal tunnel mean scores in patients $(n=11)$.

\begin{tabular}{lll}
\hline Scale & Pre-operative scores & Four-week post-operative scores \\
Symptom severity & $3.08(2-3.91)$ & $1.3(1.18-2.27)$ \\
Functional status & $3.09(1.125-4.25)$ & $1.96(1-3.13)$ \\
\hline
\end{tabular}


One weakness of our study is the group of the patients is small. In addition, the follow up may be too short. However, for a preliminary report, the result is quite encouraging, i.e., the procedure improves the symptom of the patients even in short term follow-up and improve the cosmetic appearance compare to a long incision. We hope to collect more patients and carry out longer follow-up in the future.

\section{Conclusion}

Mini-incision carpal tunnel release can be used to treat carpal tunnel syndrome that does not respond to conservative treatment with good results in both symptomatic improvement and surgical scar cosmetic.

\section{Acknowledgement}

The authors have no conflict of interest to report.

\section{References}

1. Phalen GS. The carpal-tunnel syndrome. Seventeen years' experience in diagnosis and treatment of 654 hands. J Bone Joint Surg. 1966; 48:211-28.

2. Akelman E, Weiss AC. Carpal tunnel syndrome: Results of surgical decompression. Lancet. 1969; 3: 918-9.

3. MacDonald RI, Lichtman JJ, Hanlon JN. Complications of surgical release for carpal tunnel syndrome. J Hand Surg. 1978; 3:70-6.

4. Okutsu I, Ninomiya S, Takatori Y. Endoscopic management of carpal tunnel sundrome. Arthroscopy. 1989; 5:11-8.

5. Agee JM, McCarroll HR, Tortosa RD. Endoscopic release of the carpal tunnel: A randomized prospective multicenter study. J Hand Surg.1992; 17:987-95.

6. Agee JM, Peimer CA, Pyrek JD. Endoscopic carpal tunnel release: a prospective study of complications and surgical experience. J Hand Surg.1995; 20:165-71.

7. Palmer AK, Toivonen DA. Complications of endoscopic and open carpal tunnel release. J Hand Surg. 1999; 24A:561-5.

8. Carter SL. A new instrument a carpal tunnel knife. J Hand Surg. 1991; 16:178-9.

9. Klein RD, Kotsis SV, Chung KC. Open carpal tunnel release using a 1-centimeter incision technique and outcomes for 104 patients. Plast Reconstr Surg. 2003; 111: 1616-22.

10. Cellocco P, Rossia C, Bizzarria F. Mini-open Blind Procedure Versus Limited Open Technique for Carpal Tunnel Release: A 30-Month Follow-Up Study. J Hand Surg.2005; 30:493-9.

11. Lee H, Jackson TA. Carpal tunnel release through a limited skin incision under direct visualization using a new instrument, the carposcope. Plast Reconstr Surg.1996; 98:313-9.

12. Abouzahr MK, Patsis MC, Chiu DT. Carpal tunnel release using limited direct vision. Plast Reconstr Surg. 1995; 95:534-8.

13. Hallock GG, Lutz DA. Prospective comparison of minimal incision "open” and two-portal endoscopic carpal tunnel release. Plast Reconstr Surg. 1995; 95: 941-6.

14. Bromley GS. Minimal-incision open carpal tunnel decompression. J Hand Surg.1994; 19:119-20.

15. Serra JM, Benito JR, Monner J. Carpal tunnel release with short incision.Plast Reconstr Surg. 1997; 99: 129-35.

16. Lee WB, Strickland JW. Safe carpal tunnel release via a limited palmar incision. Plast Reconstr Surg. 1998; 101:418-24.

17. Wilson KM. Double incision open technique for carpal tunnel release: an alternative to open release. J Hand Surg.1994; 19:907-12.

18. Levine DW, Simmons BP, Koris MJ, et al. A selfadministered questionnaire for the assessment of severity of symptoms \& functional status in carpal tunnel syndrome. J Bone Joint Surg.1993; 75:1585-92.

19. Watchmaker GP, Weber D, Mackinnon SE. Avoidance of transaction of the palmar cutaneous branch of the median nerve in carpal tunnel release. J Hand Surg. 1996; 21:644-50.

20. Atroshi I, Breidenbach WC, McCabe SJ. Assessment of the carpal tunnel outcome instrument in patients with nerve-compression symptoms. J Hand Surg. 1997; 22: 222-7.

21. Katz JN, Fossel KK, Simmons BP, et al. Symptoms, functional status and neuromuscular impairment following carpal tunnel release. J Hnad Surg. 1995; 20: 549-55. 